Thorax (1965), 20, 441.

\title{
Effect of venesection on arterial gas values and ventilatory function in patients with chronic bronchitis
}

\author{
DONALD MASSARO AND SOL KATZ \\ From the Pulmonary Disease Research Laboratory, Veterans Administration Hospital, Washington, D.C., \\ and the Department of Medicine, Georgetown University School of Medicine, Washington, D.C.
}

The physician faced with a patient seriously ill with chronic bronchitis often feels obliged to use all therapeutic measures, including those of doubtful value. When such patients have received therapy to promote bronchial cleansing, to increase airway patency, to control infection and to improve cardiac function by the use of digitalis, diuretics, and salt restriction, serious disability may still continue. In this situation additional therapeutic manœuvres are frequently attempted although the evidence for their usefulness may be tenuous. Since some of these subjects have raised haematocrits, venesection is often considered a justifiable procedure. Although much has been written about venesection, there is no unanimity about its usefulness in this situation.

In order to make a sound judgment concerning this mode of therapy one must weigh the beneficial effects of venesection against the possible dangers of this procedure, and in addition consider the consequences of allowing the haematocrit to remain raised. To assist in this evaluation it is necessary to have some knowledge of the effect of venesection, if any, on pulmonary function in these individuals.

The choice of parameters to evaluate the consequences of venesection, or various haematocrit levels, on lung function is difficult. Since the main purpose of the lung is to oxygenate the blood and remove metabolically produced $\mathrm{CO}_{2}$ without undue effort, it appeared that simple, easily reproducible tests, designed to measure the adequacy of gas exchange and the degree of mechanical impairment should be used. Thus this study is concerned with the effects of venesection, as well as the effects of naturally occurring alterations in haematocrit, on the arterial gas values and ventilatory studies of subjects with chronic lung disease, who, in the main, had haematocrits which varied between 50 and $60 \%$.

\section{METHODS AND MATERIALS}

SUBECTS The subjects were men ranging in age from 44 to 74 years, with a mean age of 60 years. All except one were followed by one physician for more than six months. All were considered to be free of any acute infection or clinical change when studied. A diagnosis of chronic bronchitis was made in each subject on the basis of the presence of a cough productive of mucoid or purulent sputum daily for more than three years, in the absence of other chronic pulmonary disease likely to produce this symptom. Emphysema was tentatively diagnosed in all subjects on the basis of the clinical, radiological, and pulmonary function studies.

PULMONARY FUNCTION STUDIES Oxygen uptake and carbon dioxide output were calculated using the values from the Scholander analysis of expired air and the measured expired volume (Scholander, 1947). From these values the respiratory quotient, minute ventilation, and physiological dead space were calculated. The vital capacity, timed vital capacity, and maximum breathing capacity were determined using a Wedge spirometer and a two-channel recorder. The functional residual capacity was determined by the open-circuit nitrogen washout technique (Darling, Cournand, and Richards, 1940).

Arterial gas analysis consisted of the determination of the oxygen capacity and content in the Van Slyke apparatus and a calculation of the oxygen saturation (Van Slyke and Neill, 1924). Oxygen tension was determined directly with the Clark electrode using the adaptation of Severinghaus and Bradley (1958), while the carbon dioxide tension was determined using the Severinghaus $\mathrm{PCO}_{2}$ electrode and an Instrumentation Laboratories meter. The $p \mathrm{H}$ was measured using a glass electrode in a Metrohm compensator. All gas tension and $p \mathrm{H}$ determinations were carried out at $37^{\circ} \mathrm{C}$. in duplicate.

The alveolar ventilation ( $\dot{V})$ was calculated from the $\dot{\mathrm{V}}_{\mathrm{CO}_{2}}$ and $\mathrm{PaCO}_{2}$, whereas dead space ventilation $\left(\dot{V}_{\mathrm{D}}\right)$ represented the difference between the measured minute ventilation $\left(\dot{\mathbf{V}}_{\mathbf{E}}\right)$ and the calculated $\dot{\mathrm{V}}_{\mathbf{A}}$. The alveolar oxygen tension was calculated from the 
alveolar air equation and was used to arrive at the alveolar-arterial (A-a) gradient.

The red cell mass was determined using ${ }^{51} \mathrm{Cr}$ and a figure of 0.9 to correct the venous haematocrit as previously described by Read (1954). Venous blood for haematocrit determination was placed in heparinized capillary tubes and spun in a microhaematocrit centrifuge for 10 minutes.

PLAN OF STUDY Ten studies before and after venesection were performed on eight different subjects. About half the subjects were studied as out-patients, while the remainder were electively admitted to hospital for the procedures but were maintained on the same medications as they had had before admission. The subjects were brought to the cardiopulmonary laboratory fasting, without premedication, having refrained from smoking for eight hours. A Cournand needle was placed in the brachial artery and the pulmonary function studies were performed. About $40 \mathrm{ml}$. of arterial blood was removed. Four hours later blood volume studies were performed, and the subject was then bled over a two-day period beginning the day after the initial pulmonary function studies. Blood, 400 to $1,000 \mathrm{ml}$., was removed (Table I). On the second or third day after the last venesection the pulmonary function and blood volume studies were repeated. Three of the patients studied had, in addition to the more acute studies, several blood volume or haematocrit determinations and pulmonary function tests performed over several months.

\section{RESULTS}

The oxyyen saturation fell in six subjects and rose in four after venesection. The $p \mathrm{H}$ was unchanged in eight subjects, fell in one, and rose in the other. Oxygen tension fell four times, rose on five occasions, and was unchanged in one subject. A rise in $\mathrm{PaCO}_{2}$ was noted on three occasions, a fall twice, while no significant change occurred in five experiments. The A-a gradient narrowed in six patients, widened in one, and was essentially un- changed in three patients (Tables I, II, and III). None of these individual parameters, when considered before and after venesection for the entire group (Table IV), changed significantly.

The respiratory quotients indicated a fairly steady state before and after venesection. Seven subjects exhibited a rise in respiratory equivalent, and a fall or no change occurred in three after venesection. The expired volume rose in seven subjects and fell in three, and alveolar ventilation rose in three and fell in seven patients. Dead space ventilation rose in seven subjects and fell in three, and the physiological dead space rose in six and fell in four patients. The $\dot{V}_{D} / \dot{V}_{E}$ ratio rose in eight subjects and fell in two. When each of these parameters was considered for the entire group (Table IV) before and after venesection no statistically significant changes were noted.

Lung volume and dynamic ventilatory studies (Tables III and IV), although showing some variation before and after venesection, also failed to show any consistent or significant changes. In those subjects studied both acutely and over several months (Table V) no consistent trend was noted between alterations in haematocrit and pulmonary function.

\section{DISCUSSION}

When considered apart from its effects on pulmonary function, as indicated by arterial gases and ventilatory tests, a raised haematocrit seems to impose certain physiological disadvantages on the subject. Thus when the haematocrit is raised, especially above $60 \%$, an increase in blood viscosity is known to occur (Burch and De Pasquale, 1963). Richardson and Guyton (1959) showed that, although there was no significant change in dogs in right atrial and mean pulmonary artery pressure, a fall in cardiac output associated

T A B L E I

PATIENTS STUDIED BEFORE AND AFTER VENESECTION

\begin{tabular}{|c|c|c|c|c|c|c|c|c|c|c|c|}
\hline \multirow[b]{2}{*}{ Paticnt } & \multirow{2}{*}{$\begin{array}{c}\text { Age } \\
\text { (yrs) }\end{array}$} & \multirow{2}{*}{$\begin{array}{c}\text { Height } \\
\text { (cm.) }\end{array}$} & \multirow{2}{*}{$\begin{array}{l}\text { Neight } \\
\text { (kg.) }\end{array}$} & \multirow{2}{*}{$\underset{\left(\mathrm{m} .{ }^{2}\right)}{\text { B.S.A. }}$} & \multicolumn{3}{|c|}{ Before Vcnesection } & \multirow{2}{*}{$\begin{array}{l}\text { Blood } \\
\text { Withdrawn } \\
\text { (ml.) }\end{array}$} & \multicolumn{3}{|c|}{ After Venesection } \\
\hline & & & & & $\underset{\text { (ml./kg.) }}{\text { R.C.M. }}$ & $\underset{\text { (ml./kg.) }}{\text { P.V. }}$ & $\begin{array}{l}\text { Hct } \\
(\%)\end{array}$ & & $\underset{\text { (ml./kg.) }}{\text { R.C.M. }}$ & $\underset{\text { (ml./kg.) }}{\text { P.V. }}$ & $\begin{array}{l}\text { Hct } \\
(\%)\end{array}$ \\
\hline $\begin{array}{l}\text { T.I. } \\
\text { C.G. } \\
\text { D.C. } \\
\text { R.M. } \\
\text { S.I. } \\
\text { H.C. } \\
\text { J.M. } \\
\text { C.E. } \\
\text { T.L. } \\
\text { C.G. }\end{array}$ & $\begin{array}{l}63 \\
67 \\
74 \\
71 \\
45 \\
58 \\
44 \\
62 \\
63 \\
67\end{array}$ & $\begin{array}{l}168 \\
168 \\
163 \\
173 \\
181 \\
181 \\
165 \\
173 \\
168 \\
168\end{array}$ & $\begin{array}{l}67 \cdot 0 \\
61 \cdot 0 \\
69 \cdot 0 \\
73 \cdot 0 \\
73 \cdot 0 \\
85 \cdot 0 \\
62 \cdot 3 \\
74 \cdot 0 \\
64 \cdot 0 \\
61 \cdot 0\end{array}$ & $\begin{array}{l}1.76 \\
1.69 \\
1.73 \\
1.86 \\
1.93 \\
2.06 \\
1.68 \\
1.88 \\
1.73 \\
1.69\end{array}$ & $\begin{array}{l}28 \cdot 3 \\
24 \cdot 0 \\
33 \cdot 0 \\
35 \cdot 0 \\
30 \cdot 2 \\
23 \cdot 0 \\
36 \cdot 0 \\
39 \cdot 3 \\
43 \cdot 0\end{array}$ & $\begin{array}{l}27 \cdot 9 \\
30 \cdot 0 \\
33 \cdot 0 \\
38 \cdot 0 \\
30 \cdot 2 \\
25 \cdot 0 \\
31 \cdot 0 \\
26 \cdot 2 \\
43 \cdot 0\end{array}$ & $\begin{array}{l}56.0 \\
63.0 \\
49.7 \\
56.0 \\
53.0 \\
57.0 \\
52.5 \\
59.5 \\
66.9 \\
55.0\end{array}$ & $\begin{array}{r}400 \\
1,000 \\
600 \\
600 \\
1,000 \\
1,000 \\
500 \\
500 \\
800 \\
1,000\end{array}$ & $\begin{array}{l}15.9 \\
29 \cdot 5 \\
\\
22.9 \\
17 \cdot 0 \\
31 \cdot 0 \\
33.9 \\
37 \cdot 0\end{array}$ & $\begin{array}{l}25 \cdot 2 \\
32 \cdot 0 \\
26.9 \\
31 \cdot 0 \\
34 \cdot 0 \\
29 \cdot 0 \\
37 \cdot 0\end{array}$ & $\begin{array}{l}50.0 \\
55.0 \\
43.0 \\
53.0 \\
50.0 \\
51.5 \\
39.0 \\
53.0 \\
59.8 \\
55.0\end{array}$ \\
\hline
\end{tabular}

R.C.M.,P.V., and Hct indicate the red cell mass, plasma volume, and haematocrit 
TABLE I I

EFFECT OF VENESECTION ON ARTERIAL GASES, RESPIRATORY GAS EXCHANGE, AND ALVEOLAR RESPIRATORY

\begin{tabular}{|c|c|c|c|c|c|c|c|c|c|c|c|c|c|c|c|}
\hline \multicolumn{2}{|c|}{ Patient } & $\begin{array}{l}\mathrm{SaO}_{2} \\
(\%)\end{array}$ & $\begin{array}{l}\mathrm{Pao}_{\mathbf{2}} \\
\text { (mm. } \\
\mathrm{Hg})\end{array}$ & $p \mathbf{H}$ & $\underset{\mathbf{H g})}{\mathbf{P a c o}_{\mathbf{2}}}$ & $\begin{array}{l}\dot{V}_{2} \\
\text { (ml.!) } \\
\text { min.) }\end{array}$ & $\begin{array}{l}\dot{\mathrm{Vco}}, \\
\text { (ml. } \\
\text { min.) }\end{array}$ & R.Q. & $\begin{array}{l}\text { Resp. } \\
\text { Equiv. }\end{array}$ & 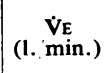 & $\mid \begin{array}{c}\dot{\mathbf{V}}_{\mathbf{A}} \\
(1 . / \mathrm{min} .)\end{array}$ & $\underset{\text { (1. } / \mathrm{min} .)}{\dot{\mathrm{V}}}$ & $\begin{array}{l}\text { D.S. } \\
\text { (ml.) }\end{array}$ & $\dot{\mathbf{V}}_{\mathbf{D}} / \dot{\mathbf{V}}_{\mathbf{E}}$ & $\begin{array}{c}\mathbf{A}-\mathbf{a} \\
(\mathrm{mm} . \\
\mathbf{H g})\end{array}$ \\
\hline \multirow{2}{*}{ T.L. } & Before & 73.8 & 44 & $7 \cdot 28$ & 78 & 217 & 182 & 0.84 & $32 \cdot 7$ & $7 \cdot 10$ & 2.00 & 5.07 & 226 & 0.72 & 14 \\
\hline & After & $71 \cdot 8$ & 40 & $7 \cdot 29$ & 83 & 197 & 229 & 0.87 & $34 \cdot 2$ & $7 \cdot 83$ & 2.04 & $5 \cdot 78$ & 222 & 0.75 & 17 \\
\hline \multirow{2}{*}{ C.G. } & Before & 84.0 & 53 & $7 \cdot 33$ & 62 & 263 & 216 & 0.81 & $27 \cdot 0$ & 7.02 & 3.00 & 4.02 & 235 & 0.56 & 27 \\
\hline & After & 83.0 & 57 & $7 \cdot 29$ & 57 & 206 & 176 & 0.86 & $36 \cdot 0$ & $7 \cdot 33$ & $2 \cdot 67$ & $4 \cdot 66$ & 228 & 0.64 & 20 \\
\hline \multirow{2}{*}{ D.C. } & Before & $92 \cdot 1$ & 68 & $7 \cdot 44$ & 50 & 221 & 175 & 0.79 & $37 \cdot 0$ & 8.05 & 3.05 & 5.00 & 229 & 0.62 & 32 \\
\hline & After & 94.5 & 80 & $7 \cdot 42$ & 52 & 184 & 153 & 0.84 & 39.0 & 8.77 & 2.07 & 4.61 & 212 & 0.65 & 10 \\
\hline \multirow{2}{*}{ R.M. } & Before & $82 \cdot 8$ & 62 & $7 \cdot 37$ & 62 & 192 & 172 & 0.90 & 31.8 & $6 \cdot 10$ & $2 \cdot 39$ & 3.71 & 240 & 0.61 & 19 \\
\hline & After & $84 \cdot 7$ & 57 & $7 \cdot 38$ & 54 & 198 & 172 & 0.86 & 33.4 & 6.09 & 2.63 & $3 \cdot 87$ & 250 & 0.59 & 31 \\
\hline \multirow{2}{*}{ S.L. } & Before & $80 \cdot 1$ & 58 & $7 \cdot 39$ & 51 & 237 & 273 & 0.87 & $29 \cdot 0$ & 7.91 & 4.01 & $3 \cdot 88$ & 222 & 0.49 & 35 \\
\hline & After & 78.7 & 49 & $7 \cdot 38$ & 54 & 225 & $2 € 6$ & 0.84 & $30 \cdot 0$ & 7.95 & 3.54 & 4.41 & 230 & 0.57 & 37 \\
\hline \multirow{2}{*}{ H.C. } & Before & $84 \cdot 5$ & 52 & $7 \cdot 33$ & 54 & 309 & 259 & 0.84 & $31 \cdot 5$ & $9 \cdot 72$ & $4 \cdot 16$ & $5 \cdot 56$ & 178 & 0.58 & 35 \\
\hline & After & $80 \cdot 8$ & 57 & $7 \cdot 32$ & 59 & 340 & 283 & 0.84 & $31 \cdot 0$ & $10 \cdot 40$ & $4 \cdot 14$ & $6 \cdot 22$ & 197 & $0 \cdot \epsilon 0$ & 24 \\
\hline \multirow{2}{*}{ J.M. } & Before & 91.4 & 68 & $7 \cdot 42$ & 45 & 204 & 165 & 0.81 & 35.4 & $7 \cdot 20$ & $3 \cdot 16$ & 4.04 & 262 & 0.57 & 27 \\
\hline & After & $95 \cdot 1$ & 69 & $7 \cdot 41$ & 47 & 187 & 154 & 0.83 & 38.5 & $7 \cdot 15$ & $2 \cdot 86$ & $4 \cdot 29$ & 296 & $0 \cdot \epsilon 0$ & 23 \\
\hline \multirow{2}{*}{ C.E. } & Before & $90 \cdot 5$ & 56 & $7 \cdot 36$ & 52 & 278 & 242 & 0.87 & 33.8 & 10.59 & 4.02 & $5 \cdot 31$ & 109 & 0.57 & 34 \\
\hline & After & 85.8 & 49 & $7 \cdot 39$ & 54 & 252 & 205 & 0.82 & $33 \cdot 2$ & $8 \cdot 34$ & $3 \cdot 28$ & 5.05 & 216 & 0.61 & 37 \\
\hline \multirow{2}{*}{ T.L. } & Before & 83.4 & 53 & $7 \cdot 34$ & 61 & 201 & 166 & 0.83 & 38.5 & $7 \cdot 15$ & $2 \cdot 21$ & 5.07 & 235 & 0.73 & 27 \\
\hline & After & $85 \cdot 2$ & 56 & $7 \cdot 34$ & 63 & 206 & 165 & 0.81 & 35.6 & $7 \cdot 28$ & $2 \cdot 28$ & 5.00 & 218 & 0.69 & 19 \\
\hline \multirow{2}{*}{ C.G. } & Before & 85.0 & 52 & $7 \cdot 31$ & 49 & 201 & 165 & 0.83 & 36.0 & $7 \cdot 12$ & 2.91 & $4 \cdot 21$ & 203 & 0.59 & 39 \\
\hline & After & $84 \cdot 0$ & 59 & $7 \cdot 32$ & 49 & 194 & 162 & 0.84 & 37.0 & $7 \cdot 13$ & $2 \cdot 88$ & $4 \cdot 75$ & 227 & $0 . C 0$ & 30 \\
\hline
\end{tabular}

R.Q. = respiratory quotient $;$ D.S. $=$ dead space

with normovolaemic polycythaemia resulted in a marked increase in pulmonary vascular resistance. In addition these authors found that when the maximum number of red cells available each minute for oxygen transport (the product of the cardiac output per kilogram and the haematocrit) is determined, this value is at a maximum at a haematocrit of 40 . This haematocrit value was within $0.6 \%$ of the mean control haematocrit in the dogs studied. Howarth, McMichael, and Sharpey-Schafer (1947) found in subjects with chronic heart failure due to pulmonary disease that an immediate reduction in right atrial and pulmonary artery pressure as well as cardiac output follows venesection. Auchincloss and Duggan (1957) made similar observations. However, because studies of pulmonary haemodynamics repeated over a prolonged period of time are not available, the duration of these changes is unknown. Since several subjects in the present study had a rather rapid re-expansion of their blood volume, mainly through a rapid rise in plasma volume, it would appear, in subjects in whom viscosity is not an important factor, that these haemodynamic changes will be short-lived. Hecht, Gaylor, and Stein (1955) found that venesection had no predictable effect on the vascular alterations of cor pulmonale. From the studies just cited it appears that a lowering of markedly raised haematocrits might improve pulmonary circulatory function. It is unlikely that the correction of moderately raised haematocrits in the 50 to $60 \%$ range would be of significant benefit for any significant length of time.

Stroebel, Hall, and Pease (1951) have shown that subjects with polycythaemia vera are predisposed to angina pectoris and myocardial infarction. However, these subjects frequently have thrombocytosis which complicates the situation. Burch and De Pasquale (1963) have called attention to the higher haematocrit levels in subjects with acute myocardial infarction as compared with agematched controls. These authors have tentatively suggested, although published controlled studies are not available, that venesection should be used in patients with erythrocytosis and symptomatic 
TABLE I I I

EFFECT OF VENESECTION ON STATIC AND DYNAMIC LUNG VOLUMES

\begin{tabular}{|c|c|c|c|c|c|c|c|}
\hline \multicolumn{2}{|c|}{ Patient } & \multirow{3}{*}{$\begin{array}{c}\begin{array}{c}\text { T.L.C. } \\
\text { (mi.) }\end{array} \\
6,055\end{array}$} & \multirow{3}{*}{$\begin{array}{l}\begin{array}{l}\text { V.C. } \\
\text { (ml.) }\end{array} \\
1,232 \\
1,513\end{array}$} & \multirow{3}{*}{$\begin{array}{c}\begin{array}{c}\text { F.R.C. } \\
(\mathrm{ml} .)\end{array} \\
5,097\end{array}$} & \multirow{3}{*}{$\frac{\begin{array}{l}\text { R.V. } \\
\text { (ml.) }\end{array}}{4,823}$} & \multirow{3}{*}{$\begin{array}{c}\begin{array}{c}\text { M.B.C. } \\
\text { (1. min.) }\end{array} \\
18 \cdot 0 \\
14 \cdot 7\end{array}$} & \multirow{3}{*}{ 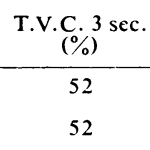 } \\
\hline T I & Before & & & & & & \\
\hline & After & & & & & & \\
\hline \multirow{2}{*}{ C.G. } & Before & & 1,860 & & & $22 \cdot 0$ & 57 \\
\hline & After & & 1,790 & & & $35 \cdot 0$ & 55 \\
\hline \multirow{2}{*}{ D.C. } & Before & & 1,495 & & & $21 \cdot 5$ & 76 \\
\hline & After & & 1,899 & & & $25 \cdot 3$ & 63 \\
\hline \multirow{2}{*}{ R.M. } & Before & & 2,040 & & & $17 \cdot 6$ & 46 \\
\hline & After & & 1,850 & & & $19 \cdot 8$ & 54 \\
\hline \multirow{2}{*}{ S. L. } & Before & 9,140 & 2,610 & 7,240 & 6,640 & $37 \cdot 0$ & 71 \\
\hline & After & 7,930 & 2,400 & 6,420 & 5,520 & $57 \cdot 0$ & 74 \\
\hline \multirow{2}{*}{ H.C. } & Before & 4,085 & 1,817 & 2,785 & 2,239 & $19 \cdot 4$ & 65 \\
\hline & After & 3,700 & 1,765 & 2,488 & 1,935 & $21 \cdot 3$ & 66 \\
\hline \multirow{2}{*}{ J.M. } & Before & & 1,620 & & & $15 \cdot 1$ & 41 \\
\hline & After & & 1,460 & & & $15 \cdot 5$ & 43 \\
\hline \multirow{2}{*}{ C.E. } & Before & & 2,170 & & & $44 \cdot 3$ & 64 \\
\hline & After & & 2,760 & & & $51 \cdot 3$ & 51 \\
\hline \multirow{2}{*}{ T.L. } & Before & & 1,332 & 3,809 & & $18 \cdot 7$ & 62 \\
\hline & After & 6,171 & 1,486 & 4,796 & 4,685 & $21 \cdot 2$ & 59 \\
\hline \multirow{2}{*}{ C.G. } & Before & 4,120 & 1,170 & & 2,970 & 14.9 & 68 \\
\hline & After & 4,490 & 1,220 & & 3,270 & $17 \cdot 2$ & 82 \\
\hline
\end{tabular}

T. L.C. = total lung capacity; V.C. = vital capacity; F.R.C. = functional residual capacity; R.V. = residual volume; M.B.C. = maximum breathing capacity; T.V.C. 3 sec. $=$ timed vital capacity third second

TABLE IV

\begin{tabular}{|c|c|c|c|}
\hline Parameter & $\begin{array}{c}\text { Mean Value } \\
\text { Pre- } \\
\text { venesection }\end{array}$ & $\begin{array}{c}\text { Mean Value } \\
\text { Post- } \\
\text { venesection }\end{array}$ & $\mathbf{p}^{1}$ \\
\hline $\begin{array}{l}\mathrm{O}_{2} \text { saturation (\%) } \\
\mathrm{PaCO}_{2}(\mathrm{~mm} \text {. Hg) } \\
\mathrm{PaO}_{2} \text { (mm. Hg) } \\
\text { pH }\end{array}$ & $\begin{array}{l}84 \cdot 8 \\
56 \cdot 4 \\
56 \cdot 6 \\
7 \cdot 36 \\
28 \cdot 9 \\
33 \cdot 3 \\
3 \cdot 09 \\
4 \cdot 59 \\
7 \cdot 80 \\
0 \cdot 60 \\
0 \cdot 214 \\
1 \cdot 73 \\
22 \cdot 8 \\
60 \cdot 2\end{array}$ & $\begin{array}{l}84 \cdot 4 \\
57 \cdot 2 \\
57 \cdot 3 \\
7 \cdot 35 \\
24 \cdot 8 \\
34 \cdot 8 \\
2 \cdot 84 \\
4 \cdot 86 \\
7 \cdot 83 \\
0 \cdot 63 \\
0 \cdot 230 \\
1 \cdot 81 \\
27 \cdot 8 \\
59 \cdot 9\end{array}$ & $\begin{aligned} &> 0.9 \\
& 0.9 \\
& 0.9 \\
& 0.9 \\
& 0.3 \\
& 0.3 \\
& 0.4 \\
& 0.3 \\
&>0.9 \\
& 0.3 \\
& 0.3 \\
& 0.8 \\
& 0.4 \\
&>0.9\end{aligned}$ \\
\hline
\end{tabular}

${ }^{1}$ P value obtained from the standard deviation of the difference of the means

ischaemic heart disease. It is curious and of interest that, while erythrocytosis of a mild to moderate degree is common in subjects with chronic lung disease, myocardial infarction is uncommon (Nonkin, Dick, and Baum, 1964).

In attempting to evaluate the effects of venesection on pulmonary function in subjects with chronic lung disease several things may be considered. First, subjects with polycythaemia vera, in the absence of intrinsic lung disease or damage to the medullary respiratory centre, rarely have abnormal ventilatory function or gas exchange (Ratto, Briscoe, Morton, and Comroe, 1955). Secondly, since the raised haematocrit is presumably a compensatory mechanism to provide additional haemoglobin for oxygen transport, could its reduction adversely affect gas exchange ? Evidence is available to indicate that correction of anaemia results in an increase in the diffusing capacity of the lung of human subjects (Rankin, McNeill, and Forster, 1961). Mochizuki, Anso, Goto, Hamamoto, and Makiguchi (1958) found in dogs that the calculated diffusing capacity of the lung for oxygen increased proportionately to an increase in haematocrit when other conditions remained constant. Burrows and Niden (1963) reported that haemorrhagic shock induced a marked fall in pulmonary diffusing capacity for carbon monoxide in dogs. Anderson and Gray (1962) noted a fall in the diffusing capacity for carbon monoxide when normovolaemic polycyth- 


\begin{tabular}{|c|c|c|c|c|}
\hline \multirow{19}{*}{ 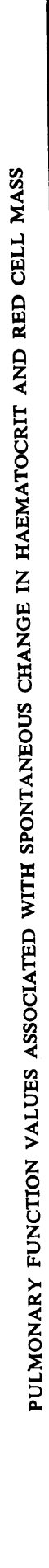 } & $\underset{\dot{\varphi}}{\stackrel{\dot{\varphi}}{\leftrightarrow}}$ & 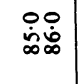 & 郘 & $\stackrel{\mathscr{\pi}}{\stackrel{\sim}{\pi}}$ \\
\hline & 离 & 弚安 & 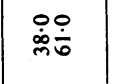 & $\stackrel{\infty}{\dot{m}}$ \\
\hline & 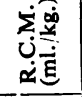 & mo & نेंn & 㐫 \\
\hline & 矛。 & ㅇํำ & $\mid$ & 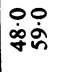 \\
\hline & 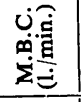 & 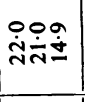 & 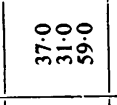 & 这 \\
\hline & $\stackrel{\dot{j}}{>}$ & 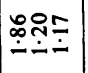 & $\mid$ & ஸึّ̆ \\
\hline & 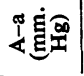 & సत्रूल & 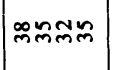 & 赵 \\
\hline & $\dot{\dot{\varphi}} \hat{\dot{\vec{E}}}$ & 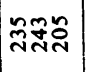 & สีสก & 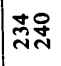 \\
\hline & $\begin{array}{l}\stackrel{\Delta}{\circ} \\
\vec{\circ}\end{array}$ & 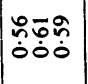 & 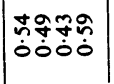 & ơ \\
\hline & 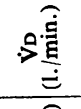 & 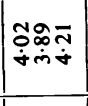 & 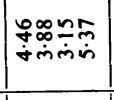 & 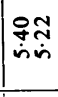 \\
\hline & 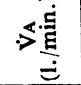 & 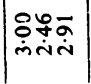 & 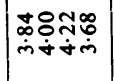 & प̄oू \\
\hline & 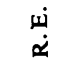 & సినిల్ల & 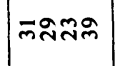 & గొల్ల \\
\hline & $\underset{\dot{\alpha}}{\dot{\alpha}}$ & 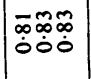 & 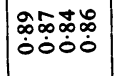 & $\begin{array}{l}T_{0}-0 \\
00 \\
00\end{array}$ \\
\hline & ठृّ & ชูตร & ถัทั์ & ide? \\
\hline & 窝 & $\underset{\dot{m}}{\dot{m}}$ & 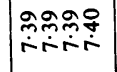 & 冓足 \\
\hline & 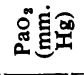 & nีกับ & 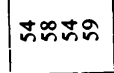 & ถูก \\
\hline & ซ్ఞొอ & 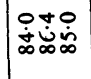 & 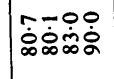 & $\begin{array}{l}\dot{p r} \\
\dot{\alpha} \tilde{\alpha} \\
\dot{\alpha}\end{array}$ \\
\hline & 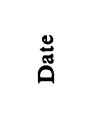 & 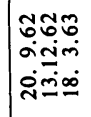 & 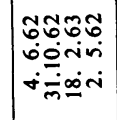 & 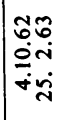 \\
\hline & 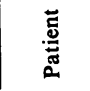 & ن் & $\dot{\dot{s}}$ & نُ \\
\hline
\end{tabular}


aemia was induced in dogs. However, in this experiment the polycythaemia was associated with a fall in cardiac output.

Thus, although it seems well documented that the diffusing capacity is directly related to the haematocrit, the extent to which this becomes a practical consideration in making a decision to lower the haematocrit in subjects with chronic lung disease is limited. Indeed, if polycythaemia is associated with a smaller cardiac output than would be present at a lower haematocrit, the pulmonary diffusing capacity might rise with a fall in haematocrit. Other alterations, in addition to changes in diffusion, are probably brought into play by venesection. Changes in ventilation perfusion relationships and the degree of venous admixture would be expected to take place if alterations in cardiac output were produced.

Although all these changes probably do occur in a complex fashion, their ultimate effect on gas exchange seems to be minimal. Thus Auchincloss and Duggan (1957) found in rather severely polycythaemic subjects with chronic lung disease little change in arterial gas values after venesection. The present study confirms these findings in a group of patients seen more commonly, those whose haematocrit is between 50 and $60 \%$ due to the hypoxaemia of chronic lung disease. Likewise, the lack of any significant changes in the $p \mathbf{H}$ of the arterial blood after venesection in both studies indicated the ability of these subjects to compensate for the loss of some buffering material.

Although difficult to evaluate and fraught with emotional overtones, the patient's subjective response to venesection is of interest. Most subjects with very high haematocrits generally feel better after venesection. The subjects in this study whose haematocrits were only moderately raised, uniformly felt more weak and tired after as compared with before venesection.

\section{SUMMARY AND CONCLUSIONS}

The effect of venesection on pulmonary gas exchange and ventilatory function was studied in eight subjects with chronic bronchitis. No \& significant changes were found after venesection. Spontaneous fluctuations in haematocrit or red cell mass were also unassociated with significant changes in arterial gas tensions, $p \mathrm{H}$ or ventilatory function.

\section{REFERENCES}

Anderson, C. B., and Gray, F. D. (1962). The circulatory and ventilatory effects of normovolemic polycythemia. Yale J. Biol. Med., 35,233 .

Auchincloss, J. H., and Duggan, J. J. (1957). Effects of venesection on pulmonary and cardiac function in patients with chronic pulmonary emphys $\epsilon$ ma and secondary polycythemia. Amer. $J$. Med., 22, 74.

Burch, G. E., and De Pasquale, N. P. (1963). Phlebotomy, Arch. intern. Med., 111, 687 .

Burrows, B and Niden, A. H. (1963). Effects of ane1 rhagic shock on rhagic shock on pulmonary diffusion in the dog lung. J. appl. Physiol., 18, 123.

Darling, R. C., Cournand, A., and Richards, D. W., Jr. (1940), Studies on the intrapulmonary mixture of gases. III. An open circuit method for measuring residual air. J. clin. Invest., 19, 609.

Hecht, H. H., Gaylor, W., and Stein, D. (1955). Vascular adjustments after phlebotomv in polycythemic subjects. Ibid., 34, 939

Howarth, S., McMichael, J., and Sharpey-Schafer, E. P. (1947). Oै Effects of oxygen, venesection and digitalis in chronic heart o failure from disease of the lungs. Clin. Sci., 6, 187.

Mochizuki, M., Anso, T., Goto, H., Hamamoto, A., and Makiguchi, Y. (1958). The dependency of the diffusing capacity on the $\mathrm{HbO}_{2}$ saturation of the capillary blood and on anemia. Jap. J. Physiol., 8,225 .

Nonkin, P. M., Dick, M. M., and Baum, G. L. (1964). Myocardia infarction in respiratory insufficiency. Arch. intern. Med., 113, 42

Rankin, J., McNeill, R. S., and Forster, R. E. (1961). The effect of anemia on the alveolar-capillary exchange of carbon monoxide in man. J. clin. Invest., 40,1323.

Ratto, O., Briscoe, W. A., Morton, J. W., and Comroe, J. H., Jr. (1955). Anoxemia secondary to polycythemia and polycythemia secondary to anoxemia. Amer. J.Med. 19,958.

Read, R. C. (1954). Studies of red-cell volume and turnover using radiochromium; description of a new 'closed' method of redcell-volume measurement. New Engl. J. Med., 250, 1021.

Richardson, T. Q., and Guyton, A. C. (1959). Effects of polycythemia and anemia on cardiac output and other circulatory factors. Amer. J. Physiol., 197, 1167.

Scholander, P. F. (1947). Analyzer for accurate estimation of respira tory gases in one-half cubic centimeter samples. $J$. biol. Chem. 167,235 .

Severinghaus, J. W., and Bradley, A. F. (1958). Electrodes for blood $\mathrm{pO}_{2}$ and $\mathrm{pCO}_{2}$ determination. J. appl. Phvsiol., 13, 515 .

Stroebel, C. F., Hall, B. E., and Pease, G. L. (195i). Evaluation of radiophospho rus therapy in primary polycythemia. J. Amer. med. Ass., 146, 1301.

Van Slyke, D.D., and Neill, J. M. (1924). The determination of gases in blood and other solutions by vacuum extraction and manometric measurement. J. biol. Chem. 61, 523 . 